\title{
SOME REMARKS ON A PROBABILITY LIMIT THEOREM FOR CONTINUED FRACTIONS
}

\author{
JORGE D. SAMUR
}

\begin{abstract}
It is shown that if a certain condition on the variances of the partial sums is satisfied then a theorem of Philipp and Stout, which implies the asymptotic fluctuation results known for independent random variables, can be applied to some quantities related to continued fractions. Previous results on the behavior of the approximation by the continued fraction convergents to a random real number are improved.
\end{abstract}

\section{Introduction. The Invariance Principle. Bound for moments}

In this note we show that an almost sure invariance principle of Philipp and Stout [17, Theorem 7.1], a probability limit theorem whose conclusion is that the partial sums process of a given sequence of random variables can be suitably approximated by a Brownian Motion, can be applied to some quantities related to the continued fraction expansion of a random number in $(0,1)$ provided a certain asymptotic variance is strictly positive (or, equivalently, if the variances of the partial sums tend to infinity). Therefore those quantities would have an analogous probabilistic behavior to that of sequences of independent, identically distributed random variables with finite moments of order greater than two.

We recall some definitions and notations. Given $\omega \in \Omega:=(0,1) \backslash \mathbb{Q}$ let

$$
\omega=\left[0, a_{1}(\omega), a_{2}(\omega), \ldots\right]=\frac{1}{a_{1}(\omega)+\frac{1}{a_{2}(\omega)+\ldots}}
$$

(the $a_{j}$ 's are positive integers) be its (infinite) simple continued fraction expansion and denote

$$
x_{j}(\omega)=\left[a_{j}(\omega), a_{j+1}(\omega), \ldots\right]=\frac{1}{T^{j-1} \omega} \quad(j \geq 1),
$$

where $T \omega=\frac{1}{\omega}-\left[\frac{1}{\omega}\right]$ ([·] is the integer part) is the continued fraction transformation,

$$
y_{j}(\omega)=\left[a_{j}(\omega), a_{j-1}(\omega), \ldots, a_{1}(\omega)\right]=a_{j}(\omega)+\frac{1}{a_{j-1}(\omega)+\frac{1}{\cdots+\frac{1}{a_{1}(\omega)}}},
$$

Received by the editors January 1, 1995.

1991 Mathematics Subject Classification. Primary 11K50, 60F17; Secondary 11K60, 60F05, $60 \mathrm{~F} 15$.

Key words and phrases. Continued fractions, approximation by the principal convergents, almost sure invariance principle.

Some results were announced at the 8th International Conference on Probability in Banach Spaces, Brunswick, Maine, July 1991. 


$$
u_{1}=x_{1}, u_{j}=x_{j}+\frac{1}{y_{j-1}} \text { if } j \geq 2 .
$$

The $a_{j}$ 's are the partial quotients and the $x_{j}$ 's the complete quotients of the omitted number $\omega$.

Observe that $y_{j}=\frac{q_{j}}{q_{j-1}}$ and $\omega=\left[0, a_{1}, \ldots, a_{j-2}, a_{j-1}+\frac{1}{x_{j}}\right]\left(=\left[0, a_{1}+\frac{1}{x_{2}}\right]\right.$, by convention, if $j=2$ ); thus $\frac{1}{x_{j}}=T^{j-1}$ gives the remainder when $\omega$ is written as a finite continued fraction. Also, if we write $\left[0, a_{1}, \ldots, a_{j}\right]=\frac{p_{j}}{q_{j}}$ with $p_{j}, q_{j}$ relatively prime then

$$
\theta_{j}(\omega):=\left|\omega-\frac{p_{j}(\omega)}{q_{j}(\omega)}\right| q_{j}^{2}(\omega)=\frac{1}{u_{j+1}(\omega)} \quad(j \geq 0)
$$

and we have $0<\theta_{j}(\omega)<1, q_{j}(\omega) \geq 2^{\frac{j-1}{2}}$; the $\frac{p_{j}}{q_{j}}$, the principal convergents, are good rational approximations to $\omega$ and $\theta_{j}(\omega)$ is a measure of the approximation.

We will look at these quantities as random variables defined on the probability space $\Omega$ endowed with its Borel $\sigma$-algebra and the Lebesgue measure $\lambda$; then $E, \operatorname{Var}, \operatorname{Cov}$ denote the expectation, variance and covariance with respect to $\lambda$.

The limiting individual distribution of $u_{j}$ or, equivalently, that of $\theta_{j}$ was exhibited by Doeblin [5, p. 365] and, independently, in the main theorem of Knuth [13] (see [18, Lemma 4.5] for a treatment along the lines of [5]), where the following heuristic connection with a method for factoring large numbers is pointed out. Developing this method Knuth [12, pp. 380-384] considers a quadratic irrationality $\sqrt{d}$ and its complete quotients $x_{n}(\sqrt{d})$ written in the form:

$$
x_{n}(\sqrt{d})=\frac{u_{n-1}+\sqrt{d}}{v_{n}} \quad(n \geq 1)
$$

where the $v_{n}$ 's are certain integers in the interval $(0,2 \sqrt{d})$; the size of $v_{n}$ is interesting (the procedure looks for the factorization of them into given small prime factors) and is related to the size of $\theta_{n-1}(\alpha), \alpha$ being the fractional part of $\sqrt{d}$, by (see [13] and [12])

$$
\frac{v_{n}}{2 \sqrt{d}}=\theta_{n-1}(\alpha)+r_{n}, \quad\left|r_{n}\right|<\frac{1}{2 q_{n-1}^{2}(\alpha) \sqrt{d}} .
$$

Note also that $\left|r_{n}\right|<\left(2^{n-1} \sqrt{d}\right)^{-1}$ and then $\left|\sum_{1}^{n} v_{k} / 2 \sqrt{d}-\sum_{0}^{n-1} \theta_{j}(\alpha)\right|<2 / \sqrt{d}$. But since the set of quadratic irrationalities has measure zero we cannot apply directly to the $v_{n}$ 's the results on the $\theta_{n}$ 's obtained here and those that we are describing.

According to [13], H. W. Lenstra posed the problem of analyzing the sequence $\theta_{1}(\omega), \theta_{2}(\omega), \ldots$ for a single $\omega$ and conjectured that for every $z \in(0,1)$

$$
\lim _{n \rightarrow \infty} \frac{1}{n} \#\left\{j \leq n: \theta_{j}(\omega) \leq z\right\}=F(z)
$$

for almost all $\omega, F$ being the limiting distribution function of $\theta_{j}$ (see also [5, p. $365]$ ). This was proved (besides other results) by Bosma, Jager and Wiedijk [3]. 
On the other hand, Doeblin [5, p. 365] stated (in terms of $u_{j}$ ) that for some constant $m$

$$
\lim _{n \rightarrow \infty} \frac{1}{n} \sum_{j=0}^{n-1} \theta_{j}(\omega)=m
$$

for almost all $\omega$; from Remark 1.1 we see that $m=\int x F(d x)=\frac{1}{4 \log 2}$. These results can be rephrased as strong laws of large numbers for the sequence $\left\{f\left(u_{j}\right)\right\}$ with $f=\mathcal{I}_{[1 / z, \infty)}$ (the indicator function of the interval) or $f(x)=\frac{1}{x}$, respectively.

1.1. Remark. If $f:[1, \infty) \longrightarrow \mathbb{R}$ satisfies $\int_{1}^{\infty}|f(x)| x^{-2} d x<\infty$ and a Lipschitz condition, then for almost all $\omega, \frac{1}{n} \sum_{1}^{n} f\left(u_{j}(\omega)\right)$ tends to the $m$ given in (3.2) below (see Remark 3.1 for a proof). This includes the previous relation. The same result holds for $y_{j}$ and $m$ given by (3.11); for $x_{j}$ and without assuming the Lipschitz condition the result is a direct consequence of the ergodic theorem $([1$, p. 45$])$. See Corollary 1.5.

Our Proposition 1.4 gives almost sure invariance principles for $\sum_{j=0}^{n-1} \theta_{j}$ and $\#\left\{j \leq n: \theta_{j} \leq z\right\}$ (for fixed $z \in(0,1)$ ) if the condition $\sigma^{2}>0$ is satisfied in these cases. This would give more precise information on the fluctuation of these sums. We have not been able to solve this problem but we improved the above two theorems in Corollary $1.5 \mathrm{~d}$ ).

Gordin [6] has shown that the condition $\sigma^{2}>0$ holds in the case of $\sum_{1}^{n} f\left(x_{j}\right)$ for many functions $f$. By using his results we prove it when $f=\mathcal{I}_{[b, \infty)}$ (the indicator function of the interval); this completes an example in [2] (see Section 4 below).

The sequence $\left\{y_{j}\right\}$ (the ratios of the denominators of consecutive principal convergents) has been considered by Lévy [14] and Doeblin [5]. See Remark 1.3 b) and Section 4 for other references to previous work. For more results on the convergence in distribution of sums of $a_{j}, x_{j}$ and $u_{j}$, see [18] (including Poisson limit theorems and the infinite variance case) and the references therein.

For the most part of the paper we assume that $f:[1, \infty) \longrightarrow \mathbb{R}$ is either

$$
f=\mathcal{I}_{[b, \infty)} \quad \text { for some } \quad b>1
$$

or a function satisfying

$$
\begin{aligned}
& |f(x)-f(y)| \leq K|x-y| \quad(x, y \geq 1) \text { for some } K>0 \\
& \text { and } \int_{1}^{\infty}|f(x)|^{2+\delta} x^{-2} d x<\infty \text { for some } \delta>0
\end{aligned}
$$

First we show that the variance of the partial sums is almost linear.

1.2. Lemma. Assume $f$ is as in (1.1) or satisfies $\int_{1}^{\infty}|f(x)|^{2} x^{-2} d x<\infty$ and the Lipschitz condition in (1.2). There exists $\sigma^{2} \geq 0$ such that

$$
\operatorname{Var}\left(\sum_{j=1}^{n} f\left(u_{j}\right)\right)=n \sigma^{2}+O(1) \quad \text { as } n \rightarrow \infty .
$$

The same assertion holds with $y_{j}$ or $x_{j}$ in place of $u_{j}$.

1.3. Remark. a) We have $\sigma^{2}=c_{0}+2 \sum_{1}^{\infty} c_{k}$ where

$$
c_{k}=\lim _{n \rightarrow \infty} \operatorname{Cov}\left(f\left(u_{n}\right), f\left(u_{n+k}\right)\right)=c_{k}^{\prime}-m^{2} \quad(k \geq 0)
$$


with $c_{k}^{\prime}$ and $m$ given by (3.4), (3.6) and (3.2), respectively. A similar remark applies to the case of $y_{j}$ and $x_{j}$ (see (3.13), (3.15), (3.11) for $y_{j}$ and $f$ satisfying (1.2); (3.19) and $(2.1)$ for $y_{j}$ when $f$ is given by (1.1); (3.20) and (3.21) for $\left.x_{j}\right)$.

b) The assertion of this lemma for the case of $x_{j}$ (note that $\left\{f\left(x_{j}\right)\right\}$ is stationary under Gauss' measure $\left.P(d \omega)=((\log 2)(1+\omega))^{-1} d \omega\right)$ is a standard fact (see [5], $[6],[15],[16])$. For $y_{j}$ it is known when $f(x)=\log x([9])$ and for Hölder $f$ with more integrability assumed ([5], [15]).

By using the lemma the following result is obtained for $u_{j}$ and $y_{j}$ as a consequence of the proof of Theorem 7.1 of Philipp and Stout [17]. The case of the complete quotients $x_{j}$ is a direct corollary of that theorem. Note that the condition $\sigma^{2}>0$ is equivalent to the convergence to infinity of the variances of the partial sums.

1.4. Proposition. Assume $f$ is as in (1.1) or (1.2) and define the partial sums process $\{S(t): t \in[0, \infty)\}$ by

$$
S(t)=\sum_{1 \leq j \leq t}\left(f\left(u_{j}\right)-m\right), \quad j \geq 0,
$$

with $m$ given by (3.2).

If the constant $\sigma^{2}$ of Lemma 1.2 is strictly positive then the almost sure invariance principle holds for $\{S(t)\}$, that is, there exists a probability space and processes $\left\{S^{*}(t): t \in[0, \infty)\right\},\{X(t): t \in[0, \infty)\}$ defined on it such that

i) $\left\{S^{*}(t)\right\}$ and $\{S(t)\}$ have the same distribution,

ii) $\{X(t)\}$ is a standard Brownian motion,

iii) $\left|S^{*}(t)-X(\sigma t)\right|=O\left(t^{\frac{1}{2}-\epsilon}\right)$ almost surely as $t \rightarrow \infty$ for some $\epsilon>0$ (the constant implied by $O$ being random).

The same result holds for $y_{j}$ and $x_{j}$ (with the value of $m$ given by (3.11) or (2.1) and by (3.20), respectively).

We refer to the Introduction of [17] for the consequences derived from the approximation iii) on the asymptotic behavior of the partial sums, including both the almost everywhere convergence results (strong law of large numbers and its refinement given by the functional law of the iterated logarithm) and the theorems on the convergence in distribution (central limit theorem and its functional version) which are classical for independent random variables.

1.5. Corollary. Let $S(n)$ be as in Proposition 1.4 for $u_{j}, y_{j}$ or $x_{j}$ (with the appropriate $m$ ). Then:

a) If $f$ is as in Lemma 1.2 and $\sigma^{2}=0$ we have $\frac{1}{r(n)} S(n) \rightarrow 0$ a.e. for each positive sequence $\{r(n)\}$ such that $\sum_{n} 1 / r(n)^{2}<\infty$.

b) If $f$ is as in (1.1) or as in (1.2) and bounded, the condition $\sigma^{2}=0$ implies that $\frac{1}{(\log n)^{1+\varepsilon}} S(n) \rightarrow 0$ a.e. for every $\varepsilon>0$.

c) If $f$ is as in (1.1) or (1.2), then $S(n)=o\left((n \log n)^{\frac{1}{2}}(\log \log n)^{\frac{1}{2}+\varepsilon}\right)$ as $n \rightarrow \infty$ a.e. for every $\varepsilon>0$.

d) If $f$ is as in (1.1) or (1.2) and bounded, then $S(n)=O\left((n \log \log n)^{1 / 2}\right)$ as $n \rightarrow \infty$ a.e.

As a technical aside, we include the following lemma, which implies a convergence of moments result. 
1.6. Lemma. Write $S_{n, h}=\sum_{j=h+1}^{h+n}\left(f\left(u_{j}\right)-m\right)$ for $h \geq 0, n \geq 1$ (with $m$ given by (3.2)). Then, if $f$ satisfies (1.2) with $0<\delta \leq 1$,

$$
\sup _{n \geq 1, h \geq 0} E\left(\left|\frac{1}{\sqrt{n}} S_{n, h}\right|^{2+\delta}\right)<\infty .
$$

The same is true for $y_{j}$ and $x_{j}$.

(The proof of this lemma can be easily adapted to work in the case in which $f$ satisfies a Hölder condition provided (3.1) and (3.23) below are verified.)

\section{Preliminaries}

The application of the methods and results of [17] is based on the well known fact that the random variables $a_{j}$, under $\lambda$ or $P$ (defined in Remark $1.3 \mathrm{~b}$ ) above), have good properties of weak dependence ([10], [8], [1, p. 50], [11]); moreover, $\left\{a_{j}\right\}$ is stationary under $P$.

We refer to $[1, \S 4]$ or $[12, \S 4.5 .3]$ for the basic properties of continued fractions. We shall use that given positive integers $i_{1}, \ldots, i_{k}, k \geq 1$, and $t \in[0,1]$ we have

$$
\left[0, i_{1}, \ldots, i_{k}+t\right]=\frac{p_{k}+t p_{k-1}}{q_{k}+t q_{k-1}}
$$

where the integers $p_{h}, q_{h}$ are defined recursively by

$$
\begin{array}{lllll}
p_{0}=0, & p_{1}=1, & p_{h}=i_{h} p_{h-1}+p_{h-2} & \text { if } & 2 \leq h \leq k, \\
q_{0}=1, & q_{1}=i_{1}, & q_{h}=i_{h} q_{h-1}+q_{h-2} & \text { if } & 2 \leq h \leq k .
\end{array}
$$

Then, $p_{h-1} q_{h}-p_{h} q_{h-1}=(-1)^{h}(1 \leq h \leq k) ; p_{h}, q_{h}$ are relatively prime. We will use two results of Lévy [14] (the second one in an equivalent formulation):

2.1. Lemma. There exists $r \in(0,1)$ such that

$$
\sup _{y}\left|\lambda\left(y_{n} \leq y\right)-G(y)\right|=O\left(r^{n}\right) \quad \text { as } n \rightarrow \infty,
$$

(in the sup, $y$ varies in $\mathbb{R}) G$ being the distribution function with density

$$
\mathcal{I}_{[1, \infty)}(y)(y(y+1) \log 2)^{-1}
$$

2.2. Lemma. If $n \geq 2$ and $y=\left[k_{n-1}, \ldots, k_{1}\right]$ with $k_{1}, \ldots, k_{n-1} \in \mathbb{N}^{*}$ then

$$
\int_{\left\{y_{n-1}=y\right\}} h\left(x_{n}\right) d \lambda=\left(\int_{1}^{\infty} h(x) \frac{y(y+1)}{(x y+1)^{2}} d x\right) \lambda\left(y_{n-1}=y\right)
$$

for any Borel measurable function $h$, provided one of the two members exists. 


\section{Proofs}

Proof of Lemma 1.2. a) We deal first with $u_{j}$. Assume that $f$ satisfies (1.2).

By (2.2) we have

$$
\begin{aligned}
E f\left(u_{n}\right) & =E f\left(x_{n}+\frac{1}{y_{n-1}}\right)=\sum_{y} \int_{\left\{y_{n-1}=y\right\}} f\left(x_{n}+\frac{1}{y}\right) d \lambda \\
& =\int_{[1, \infty)} g d \mathcal{L}\left(y_{n-1}\right)
\end{aligned}
$$

where $g(y)=\int_{1}^{\infty} f\left(x+\frac{1}{y}\right) \beta(x, y) d x$ with $\beta(x, y)=y(y+1)(x y+1)^{-2}$ and $\mathcal{L}\left(y_{n-1}\right)$ denotes the law of $y_{n-1}$ (in the sum, $y$ takes all possible values of $y_{n-1}$ ). Denote by $\tilde{g}(x, y)$ the integrand in the definition of $g$. Suppose $1 \leq y<y^{\prime}$; we have

$$
\left|\tilde{g}(x, y)-\tilde{g}\left(x, y^{\prime}\right)\right| \leq 2(2|f(x)|+3 K) \frac{1}{x^{2} y^{2}}\left|y-y^{\prime}\right| \quad \text { for } x \geq 1
$$

(note that $|\partial \beta(x, y) / \partial y| \leq 4(x y)^{-2}, \beta(x, y) \leq 2 x^{-2}$ ) and then $\left|g(y)-g\left(y^{\prime}\right)\right| \leq$ $C y^{-2}\left|y-y^{\prime}\right|$ for some $C$ (depending on $f$ ). Hence $g$ is absolutely continuous with $\left|g^{\prime}(y)\right| \leq C y^{-2}$ a.e. and (2.1), through an integration by parts, gives

$$
\left|E f\left(u_{n}\right)-m\right|=O\left(r^{n}\right)
$$

with

$$
\begin{aligned}
m: & =\int_{[1, \infty)} g d G=\int_{0}^{1} \int_{0}^{1} f\left(\frac{1}{s}+t\right) \mu(d s, d t) \\
& =\int_{1}^{\infty} f(x) \frac{1}{\log 2}\left(\mathcal{I}_{[1,2]}(x) \frac{1}{x}\left(1-\frac{1}{x}\right)+\mathcal{I}_{(2, \infty)}(x) \frac{1}{x^{2}}\right) d x
\end{aligned}
$$

$\mu$ being the measure on the unit square given by

$$
\mu(d s, d t)=\frac{1}{\log 2} \frac{d s d t}{(1+s t)^{2}} .
$$

Similarly, one can show that

$$
\left|E f^{2}\left(u_{n}\right)-c_{0}^{\prime}\right|=O\left(r^{n}\right)
$$

with

$$
c_{0}^{\prime}:=\int_{0}^{1} \int_{0}^{1} f^{2}\left(\frac{1}{s}+t\right) \mu(d s, d t)
$$

and that for $k \geq 1$

$$
\left|E\left(f\left(u_{n}\right) f\left(u_{n+k}\right)\right)-c_{k}^{\prime}\right|=O\left(r^{n}\right),
$$

the constant involved in $O$ being independent of $k$, with

$$
c_{k}^{\prime}:=\int_{0}^{1} \int_{0}^{1} f\left(\frac{1}{s}+t\right) f\left(\frac{1}{T^{k}(s)}+\left[0, a_{k}(s), \ldots, a_{2}(s), a_{1}(s)+t\right]\right) \mu(d s, d t)
$$


(when $k=1$, the finite continued fraction inside the integral reduces to $\left[0, a_{1}(s)+t\right]$ ).

We prove only the second assertion. Fix $k \geq 1$. If $n \geq 1$, since

$$
x_{n+k}=\frac{1}{T^{k}\left(\frac{1}{x_{n}}\right)}=x_{k+1}\left(\frac{1}{x_{n}}\right) \text { and } \frac{1}{y_{n+k-1}}=v_{k}\left(x_{n}, y_{n-1}\right)
$$

with

$$
v_{k}(x, y)=\left[0, a_{k}\left(\frac{1}{x}\right), \ldots, a_{2}\left(\frac{1}{x}\right), a_{1}\left(\frac{1}{x}\right)+\frac{1}{y}\right],
$$

by $(2.2)$ we have

$$
\begin{aligned}
& E\left(f\left(u_{n}\right) f\left(u_{n+k}\right)\right) \\
& \quad=\sum_{y} \int_{\left\{y_{n-1}=y\right\}} f\left(x_{n}+\frac{1}{y}\right) f\left(x_{k+1}\left(\frac{1}{x_{n}}\right)+v_{k}\left(x_{n}, y\right)\right) d \lambda \\
& \quad=\int_{[1, \infty)} h_{k} d \mathcal{L}\left(y_{n-1}\right)
\end{aligned}
$$

where $h_{k}(y)=\int_{1}^{\infty} f\left(x+\frac{1}{y}\right) f\left(x_{k+1}\left(\frac{1}{x}\right)+v_{k}(x, y)\right) \beta(x, y) d x$.

Note that $v_{k}(x, y)=\frac{p_{k} y+p_{k-1}}{q_{k} y+q_{k-1}}$ where $p_{j}, q_{j}$ are defined for $a_{k}\left(\frac{1}{x}\right), \ldots, a_{1}\left(\frac{1}{x}\right)$ as in Section 2; then $\left|d v_{k}(x, y) / d y\right|=\left|(-1)^{k+1}\left(q_{k} y+q_{k-1}\right)^{-2}\right| \leq y^{-2}$. On the other hand, $0 \leq x_{k+1}\left(\frac{1}{x}\right)+v_{k}(x, y)-a_{k+1}\left(\frac{1}{x}\right) \leq 2$ which gives $\mid f\left(x_{k+1}\left(\frac{1}{x}\right)+v_{k}(x, y)\right)-$ $f\left(a_{k+1}\left(\frac{1}{x}\right)\right) \mid \leq 2 K$. Assume $1 \leq y<y^{\prime}$. For $x \geq 1$, if

$$
\tilde{h}_{k}(x, y)=\alpha_{k}(x, y) \beta(x, y) \quad(\text { say })
$$

is the integrand in the definition of $h_{k}$, we can show that

$$
\begin{aligned}
& \left|\alpha_{k}(x, y)-\alpha_{k}\left(x, y^{\prime}\right)\right| \leq\left\{|f(x)|+\left|f\left(a_{k+1}\left(\frac{1}{x}\right)\right)\right|+3 K\right\} K \frac{1}{y^{2}}\left|y-y^{\prime}\right|, \\
& \left|\alpha_{k}\left(x, y^{\prime}\right)\right| \leq\{|f(x)|+K\}\left\{\left|f\left(a_{k+1}\left(\frac{1}{x}\right)\right)\right|+2 K\right\}
\end{aligned}
$$

and then

$\left|\tilde{h}_{k}(x, y)-\tilde{h}_{k}\left(x, y^{\prime}\right)\right| \leq C\left\{|f(x)|+\left|f\left(a_{k+1}\left(\frac{1}{x}\right)\right)\right|+\left|f(x) f\left(a_{k+1}\left(\frac{1}{x}\right)\right)\right|+C^{\prime}\right\} \frac{1}{x^{2} y^{2}}\left|y-y^{\prime}\right| ;$ moreover, observe that (1.2) gives, using the stationarity of $\left\{a_{j}\right\}$ under Gauss' measure $P$ :

$$
\int_{1}^{\infty}\left|f\left(a_{k+1}\left(\frac{1}{x}\right)\right)\right| G(d x)=E_{P}\left|f\left(a_{1}\right)\right|<\infty
$$

and

$$
\int_{1}^{\infty}\left|f(x) f\left(a_{k+1}\left(\frac{1}{x}\right)\right)\right| G(d x) \leq\left(\int_{1}^{\infty} f^{2}(x) G(d x)\right)^{1 / 2}\left(E_{P}\left(f^{2}\left(a_{1}\right)\right)\right)^{1 / 2}<\infty .
$$

Hence $\left|h_{k}(y)-h_{k}\left(y^{\prime}\right)\right| \leq C^{\prime \prime} y^{-2}\left|y-y^{\prime}\right|$ with $C^{\prime \prime}$ independent of $k$. As above, this implies (3.5). 
Now consider the r.v.'s $\eta_{j}=f\left(u_{j}\right)-E f\left(u_{j}\right)(j \geq 1)$ and the $\sigma$-algebras

$$
\mathcal{M}_{j \ell}=\left\{\begin{array}{l}
\sigma\left(a_{j-\ell}, \ldots, a_{j+\ell}\right), \quad j>\ell, \\
\sigma\left(a_{1}, \ldots, a_{j+\ell}\right), \quad j \leq \ell
\end{array}\right.
$$

As in $[18$, proof of Lemma 4.3$]$ we can show that

$$
\left|\eta_{j}-E\left[\eta_{j} \mid \mathcal{M}_{j \ell}\right]\right| \leq \frac{6 K}{2^{\ell}} \quad \text { a.s. }
$$

$\left(E\left[\eta_{j} \mid \mathcal{M}_{j \ell}\right]\right.$ is the conditional expectation of $\eta_{j}$ given $\left.\mathcal{M}_{j \ell}\right)$, giving the same bound for the $L^{2}$ norm of this difference; following [2, p. 185] we obtain that $\left|E\left(\eta_{n} \eta_{n+k}\right)\right| \leq$ $A\left(r^{\prime}\right)^{k}$ for some constants $A$ and $r^{\prime} \in(0,1)$. Then if $c_{n k}:=E\left(\eta_{n} \eta_{n+k}\right)$ we have $\left|c_{n k}\right| \leq A\left(r^{\prime}\right)^{k}$ and (3.1), (3.3) and (3.5) imply that $c_{k}:=\lim _{n \rightarrow \infty} c_{n k}=c_{k}^{\prime}-m^{2}$ $(k \geq 0)$ satisfies $\left|c_{n k}-c_{k}\right| \leq A^{\prime} r^{n}$ with $A^{\prime}$ independent of $k$. From this (see $[9$, proof of Lemma 7]) we can conclude the proof when $f$ satisfies (1.2).

Assume $f$ is the indicator function in (1.1). It is sufficient to show that (3.1), (3.5) and a suitable version of (3.8) are satisfied. We prove the last two assertions.

Write $\tilde{h}_{k}(x, y)=\alpha_{k}(x, y) \beta(x, y)$ for the integrand in the $h_{k}$ considered in (3.7). Assume $1 \leq y<y^{\prime}$ and $k$ odd; then

$$
\begin{aligned}
h_{k}(y)-h_{k}\left(y^{\prime}\right)= & \int_{1}^{\infty}\left\{\alpha_{k}(x, y)-\alpha_{k}\left(x, y^{\prime}\right)\right\} \beta(x, y) d x \\
& +\int_{1}^{\infty} \alpha_{k}\left(x, y^{\prime}\right)\left\{\beta(x, y)-\beta\left(x, y^{\prime}\right)\right\} d x=I_{1}+I_{2} \quad \text { (say). }
\end{aligned}
$$

We have $\left|I_{2}\right| \leq C y^{-2}\left|y-y^{\prime}\right|$. Now consider the set

$$
A_{k}=\left\{\omega \in \Omega: b-v_{k}\left(\frac{1}{\omega}, y^{\prime}\right) \leq x_{k+1}(\omega)<b-v_{k}\left(\frac{1}{\omega}, y\right)\right\}
$$

and observe that $v_{k}(x, u)$ is increasing in $u$; then

$$
\left|I_{1}\right| \leq \int_{b-\frac{1}{y}}^{b-\frac{1}{y^{\prime}}} \beta(x, y) d x+\int_{1}^{\infty} \mathcal{I}_{A_{k}}\left(\frac{1}{x}\right) \beta(x, y) d x=I_{1}^{\prime}+I_{1}^{\prime \prime} \quad \text { (say) }
$$

We have $\left|I_{1}^{\prime}\right| \leq 2 \int_{b-\frac{1}{y}}^{b-\frac{1}{y^{\prime}}} x^{-2} d x \leq 2(b-1)^{-2} y^{-2}\left|y-y^{\prime}\right|$. From elementary properties of continued fractions, for $\omega \in \Omega$ and $u \geq 1$ we have $v_{k}\left(\frac{1}{\omega}, u\right)=\tilde{v}_{k}\left(y_{k}(\omega), u\right)$ where $\tilde{v}_{k}(z, u)$ is a function of $z \in V_{y_{k}}$, the set of values of $y_{k}$, and $u \geq 1$; moreover $\left|d \tilde{v}_{k}(z, u) / d u\right| \leq u^{-2}$. Then, using (2.2) again,

$$
\begin{aligned}
\left|I_{1}^{\prime \prime}\right| & \leq 2 \lambda\left(A_{k}\right)=2 \sum_{z \in V_{y_{k}}} \int_{\left\{y_{k}=z\right\}} \mathcal{I}_{\left[b-\tilde{v}_{k}\left(z, y^{\prime}\right), b-\tilde{v}_{k}(z, y)\right]}\left(x_{k+1}\right) d \lambda \\
& \leq 4 \sum_{z \in V_{y_{k}}}\left(\int_{b-\tilde{v}_{k}\left(z, y^{\prime}\right)}^{b-\tilde{v}_{k}(z, y)} \frac{d x}{x^{2}}\right) \lambda\left(y_{k}=z\right) \\
& =4 \sum_{z \in V_{y_{k}}} \frac{\tilde{v}_{k}\left(z, y^{\prime}\right)-\tilde{v}_{k}(z, y)}{\left(b-\tilde{v}_{k}(z, y)\right)\left(b-\tilde{v}_{k}\left(z, y^{\prime}\right)\right)} \lambda\left(y_{k}=z\right) \\
& \leq 4 \frac{1}{(b-1)^{2}} \frac{1}{y^{2}}\left|y-y^{\prime}\right| .
\end{aligned}
$$


A similar argument applies when $k$ is even and we conclude that $\left|h_{k}(y)-h_{k}\left(y^{\prime}\right)\right| \leq$ $C^{\prime} y^{-2}\left|y-y^{\prime}\right|$ with $C^{\prime}$ independent of $k$. As above, this implies (3.5).

Now consider the sets $J_{j \ell}=\left\{b-\frac{6}{2^{\ell}}<u_{j} \leq b+\frac{6}{2^{\ell}}\right\}(j \geq 1, \ell \geq 1)$. Arguing as in $\left[19\right.$, p. 906] one can show that, with the notations of $(3.8), \eta_{j}=E\left[\eta_{j} \mid \mathcal{M}_{j \ell}\right]$ a.s. on $J_{j \ell}^{c}$ and $\lambda\left(J_{j \ell}\right) \leq C 2^{-\ell}$ with $C$ depending only on $b$ (this uses $(2.2)$ ); then

$$
\left\|\eta_{j}-E\left[\eta_{j} \mid \mathcal{M}_{j \ell}\right]\right\|_{2} \leq C^{\frac{1}{2}} \frac{1}{\left(2^{\frac{1}{2}}\right)^{\ell}}
$$

b) Case $\left\{y_{j}\right\}$. Assume that $f$ satisfies (1.2). Noting that $y_{n}=\left[x_{n}\right]+y_{n-1}^{-1}$ and arguing as in a) one obtains that

$$
\left|E f\left(y_{n}\right)-m\right|=O\left(r^{n}\right)
$$

with

$$
m:=\int_{0}^{1} \int_{0}^{1} f\left(\left[\frac{1}{s}\right]+t\right) \mu(d s, d t)
$$

$(\mu$ as in $(3.2))$

$$
\left|E f^{2}\left(y_{n}\right)-c_{0}^{\prime}\right|=O\left(r^{n}\right)
$$

with

$$
\begin{gathered}
c_{0}^{\prime}=\int_{0}^{1} \int_{0}^{1} f^{2}\left(\left[\frac{1}{s}\right]+t\right) \mu(d s, d t), \\
\left|E\left(f\left(y_{n}\right) f\left(y_{n+k}\right)\right)-c_{k}^{\prime}\right|=O\left(r^{n}\right),
\end{gathered}
$$

the constant involved in $O$ being independent of $k \geq 1$, with

$$
c_{k}^{\prime}=\int_{0}^{1} \int_{0}^{1} f\left(\left[\frac{1}{s}\right]+t\right) f\left(\left[a_{k+1}(s), \ldots, a_{2}(s), a_{1}(s)+t\right]\right) \mu(d s, d t),
$$

and

$$
\left|\eta_{j}-E\left[\eta_{j} \mid \mathcal{M}_{j \ell}\right]\right| \leq \frac{6 K}{2^{\ell}} \quad \text { a.s. } \quad\left(\eta_{j}=f\left(y_{j}\right)-E f\left(y_{j}\right)\right)
$$

The proof in this case can be concluded as for $\left\{u_{j}\right\}$.

Assume $f$ is the indicator in (1.1). In this case (3.10) is (2.1). Before proving (3.14) we obtain the analog of (3.9) for $\eta_{j}=f\left(y_{j}\right)-E f\left(y_{j}\right)$. If $j \leq \ell, E\left[\eta_{j} \mid \mathcal{M}_{j \ell}\right]=\eta_{j}$ a.s.; if $j>\ell$, by considering $J_{j \ell}:=\left\{b-\frac{4}{2^{\ell}} \leq y_{j} \leq b+\frac{4}{2^{\ell}}\right\}$ we can see that $E\left[\eta_{j} \mid \mathcal{M}_{j \ell}\right]=\eta_{j}$ a.s. on $J_{j \ell}^{c}$ (because $\left|y_{j}(\omega)-y_{j}\left(\omega^{\prime}\right)\right|<4 / 2^{\ell}$ if $\omega, \omega^{\prime}$ have the same partial quotients $a_{j}, a_{j-1}, \ldots, a_{j-\ell}$; see, for example, [18, proof of Lemma 4.3]) and $\lambda\left(J_{j \ell}\right) \leq C r^{\ell}$ (by using (2.1), since $j>\ell$ and $r>\frac{1}{2}$; see [11]). Hence

$$
\left\|\eta_{j}-E\left[\eta_{j} \mid \mathcal{M}_{j \ell}\right]\right\|_{2} \leq C^{\frac{1}{2}}\left(r^{\frac{1}{2}}\right)^{\ell}
$$

with $C$ depending only on $b$. 
In order to prove (3.14) (but with $c_{k}^{\prime}$ different from those in (3.15)) we now condition with respect to $y_{n}$ :

$$
\begin{aligned}
E\left(f\left(y_{n}\right) f\left(y_{n+k}\right)\right) & =\sum_{y} \int_{\left\{y_{n}=y\right\}} f(y) f\left(\frac{1}{v_{k}\left(x_{n+1}, y\right)}\right) d \lambda \\
& =\int_{[b, \infty)} h_{k} d \mathcal{L}\left(y_{n}\right)
\end{aligned}
$$

where $h_{k}(y)=\int_{1}^{\infty} f\left(\frac{1}{v_{k}(x, y)}\right) \beta(x, y) d x$. Write $\alpha_{k}(x, y)=f\left(\frac{1}{v_{k}(x, y)}\right)$. If $b \leq$ $y<y^{\prime}$ we have as before $h_{k}(y)-h_{k}\left(y^{\prime}\right)=I_{1}+I_{2}$ with $\left|I_{2}\right| \leq C y^{-2}\left|y-y^{\prime}\right|$ and $I_{1}=\int_{1}^{\infty}\left(\mathcal{I}_{\left\{s: v_{k}(s, y) \leq \frac{1}{b}\right\}}(x)-\mathcal{I}_{\left\{s: v_{k}\left(s, y^{\prime}\right) \leq \frac{1}{b}\right\}}(x)\right) \beta(x, y) d x$. Fix an odd positive integer $k$ (we omit the proof for even $k$ ). Then $v_{k}(x, \cdot)$ is increasing and

$$
\begin{aligned}
I_{1} & =\int_{1}^{\infty} \mathcal{I}_{\left\{s: v_{k}(s, y) \leq \frac{1}{5}<v_{k}\left(s, y^{\prime}\right)\right\}}(x) \beta(x, y) d x \\
& \leq 2 \lambda\left(A_{k}\left(y, y^{\prime}\right)\right)
\end{aligned}
$$

where

$$
A_{k}\left(y, y^{\prime}\right)=\left\{\omega \in \Omega: v_{k}\left(\frac{1}{\omega}, y\right) \leq \frac{1}{b}<v_{k}\left(\frac{1}{\omega}, y^{\prime}\right)\right\}
$$

We will use the following fact, which can be easily proved by induction:

$\left(^{*}\right)$ Given positive integers $h, p>h, a_{1}, \ldots, a_{h}, b_{1}, \ldots, b_{p}$ and $\xi, \eta, \eta^{\prime} \in(0,1)$,

$$
\begin{gathered}
{\left[a_{h}, \ldots, a_{1}+\eta\right] \leq\left[b_{1}, \ldots, b_{p}+\xi\right] \leq\left[a_{h}, \ldots, a_{1}+\eta^{\prime}\right]} \\
\Longrightarrow \frac{1}{\eta^{\prime}} \leq\left[b_{h+1}, \ldots, b_{p}+\xi\right] \leq \frac{1}{\eta} \text { if } h \text { is odd } \\
\frac{1}{\eta} \leq\left[b_{h+1}, \cdots, b_{p}+\xi\right] \leq \frac{1}{\eta^{\prime}} \text { if } h \text { is even } .
\end{gathered}
$$

(Recall the convention that if, for example, $p=h+1$ then $\left[b_{h+1}, \ldots, b_{p}+\xi\right]=b_{p}+\xi$ ).

Suppose that $b$ is irrational and write $b=\left[b_{1}, \ldots, b_{k+1}+\xi\right], \xi \in(0,1)$, that is, $b_{k+1}+\xi=x_{k+1}(b)$. Using (*) (with $h=k, p=k+1$ ) we see that if $A_{k}\left(y, y^{\prime}\right) \neq \emptyset$ then $y \leq x_{k+1}(b) \leq y^{\prime}$. Moreover assume that $b<x_{k+1}(b)$; since $A_{k}\left(y, y^{\prime}\right)=\emptyset$ if either $b \leq y<y^{\prime}<x_{k+1}(b)$ or $x_{k+1}(b)<y<y^{\prime}$, we conclude that for any $c_{1}, c_{2}$ such that $b<c_{1}<x_{k+1}(b)<c_{2}, h_{k}$ is absolutely continuous and $\left|h_{k}^{\prime}(y)\right| \leq C y^{-2}$ on each of the two intervals $\left[b, c_{1}\right],\left[c_{2}, \infty\right)$. For all sufficiently large $n$ put $c_{1}(n)=$ $x_{k+1}(b)-\frac{1}{2^{n}}, c_{2}(n)=x_{k+1}(b)+\frac{1}{2^{n}}$, write

$$
\begin{aligned}
\int_{[b, \infty)} h_{k} d \mathcal{L}\left(y_{n}\right)= & \int_{\left[b, c_{1}(n)\right)} h_{k} d \mathcal{L}\left(y_{n}\right) \\
& +\int_{\left[c_{2}(n), \infty\right)} h_{k} d \mathcal{L}\left(y_{n}\right)+\int_{\left[c_{1}(n), c_{2}(n)\right)} h_{k} d \mathcal{L}\left(y_{n}\right)
\end{aligned}
$$

and consider the analogous expression for $\int_{[b, \infty)} h_{k} d G=: c_{k}^{\prime}$. By (2.1) the last term in (3.18) is bounded by $2 \lambda\left(c_{1}(n) \leq y_{n}<c_{2}(n)\right)=O\left(r^{n}\right)$ and a similar bound 
holds for the third term in the expression of $c_{k}^{\prime}$. The absolute value of the difference between the first term in (3.18) and the corresponding term of $c_{k}^{\prime}$ is bounded by

$$
\begin{aligned}
h_{k}(b) \mid & \lambda\left(y_{n} \in\left[b, c_{1}(n)\right)\right)-G\left[b, c_{1}(n)\right) \mid \\
& +\int_{b}^{c_{1}(n)}\left|h^{\prime}{ }_{k}(t)\right|\left|\lambda\left(y_{n} \in\left(t, c_{1}(n)\right)\right)-G\left(t, c_{1}(n)\right)\right| d t \\
& \leq\left(h_{k}(b)+C\left(\int_{b}^{\infty} t^{-2} d t\right)\right) O\left(r^{n}\right)=O\left(r^{n}\right) .
\end{aligned}
$$

For the difference between the second terms the bound is

$$
\begin{aligned}
h_{k}\left(c_{2}(n)\right) \mid & \lambda\left(y \geq c_{2}(n)\right)-G\left[c_{2}(n), \infty\right) \mid \\
& +\int_{c_{2}(n)}^{\infty}\left|h_{k}^{\prime}(t)\right|\left|\lambda\left(y_{n}>t\right)-G(t, \infty)\right| d t=O\left(r^{n}\right) .
\end{aligned}
$$

This proves (3.14) when $b$ is irrational and $x_{k+1}(b)>b$ with

$$
c^{\prime}{ }_{k}=\int_{0}^{1} \int_{0}^{1} \mathcal{I}_{[b, \infty)}\left(\frac{1}{t}\right) \mathcal{I}_{[b, \infty)}\left(\left[a_{k}(s), \ldots, a_{1}(s)+t\right]\right) \mu(d s, d t) ;
$$

if $x_{k+1}(b)=b$ a similar argument works by considering the intervals $\left[b, b+\frac{1}{2^{n}}\right),[b+$ $\left.\frac{1}{2^{n}}, \infty\right)$ and if $x_{k+1}(b)<b$ it is enough to consider $[b, \infty)$. Assume $b=\left[b_{1}, \ldots, b_{m}\right]$ with $m \geq 1, b_{1}, \ldots, b_{m}$ positive integers, $b_{m}>1$. If $k \leq m-1$ ( $k$ odd) and $b \leq y<y^{\prime}$ we have that $A_{k}\left(y, y^{\prime}\right) \neq \emptyset$ implies $y \leq\left[b_{k+1}, \ldots, b_{m}\right] \leq y^{\prime}$ (if $k<m-1$ use $\left(^{*}\right)$ with $h=k, p=m-1, \xi=b_{m}^{-1}$; if $k=m-1$, take $h=m-2, p=m-1$ to deduce first that if $\omega \in A_{k}\left(y, y^{\prime}\right)$ then $\left.a_{1}(\omega)+\frac{1}{y^{\prime}} \leq b_{m-1}+\frac{1}{b_{m}} \leq a_{1}(\omega)+\frac{1}{y}\right)$; we can argue as above with $x_{k+1}(b)$ replaced by $\left[b_{k+1}, \ldots, b_{m}\right]$. If $k \geq m$ we have that $A_{k}\left(y, y^{\prime}\right)=\emptyset$ when $b \leq y<y^{\prime}$ and we consider $[b, \infty)$ (suppose $k$ and $m$ odd; if $\omega \in A_{k}\left(y, y^{\prime}\right)$ and $h=m-2, p=m-1, \xi=b_{m}^{-1}$, then $\left[a_{k-(m-2)}(\omega), \ldots, a_{1}(\omega)+\right.$ $\left.\frac{1}{y}\right] \leq b_{m-1}+\xi \leq\left[a_{k-(m-2)}(\omega), \ldots, a_{1}(\omega)+\frac{1}{y^{\prime}}\right]$, so that $a_{k-(m-2)}(\omega)=b_{m-1}$ and $\left[a_{k-(m-1)}(\omega), \ldots, a_{1}(\omega)+\frac{1}{y^{\prime}}\right] \leq b_{m} \leq\left[a_{k-(m-1)}(\omega), \ldots, a_{1}(\omega)+\frac{1}{y}\right]$ which in turn implies $a_{k-(m-1)}(\omega)<b_{m}<a_{k-(m-1)}(\omega)+1$; but $b_{m}$ is an integer).

c) We omit the proof for $x_{j}$ but write out the constants:

$$
\begin{gathered}
m:=\int_{0}^{1} \int_{0}^{1} f\left(\frac{1}{s}\right) \mu(d s, d t)=\int_{1}^{\infty} f(x) \frac{1}{\log 2} \frac{d x}{x(x+1)}, \\
c_{k}^{\prime}:=\int_{0}^{1} \int_{0}^{1} f\left(\frac{1}{s}\right) f\left(\frac{1}{T^{k}(s)}\right) \mu(d s, d t) \quad(k \geq 0) .
\end{gathered}
$$

3.1. Remark. We prove the assertion in Remark 1.1 for $u_{j}$. Assume $f$ is as stated. For each $k \geq 2$ define $g_{k}=f\left(\left[a_{k}, \ldots, a_{2 k}\right]+1 / y_{k-1}\right)$ (we omit $\omega$ ). Since $\left|\left[a_{k}, \ldots, a_{2 k}\right]+1 / y_{k-1}-a_{k}\right| \leq 2$ we see that $E_{P}\left|g_{k}\right| \leq 2 K+E_{P}\left|f\left(a_{k}\right)\right|<\infty$ because $E_{P}\left|f\left(a_{k}\right)\right|=E_{P}\left|f\left(a_{1}\right)\right|$ which is finite by the properties of $f$. 
By the ergodic theorem ( $T$ is ergodic under $P$; see [1, p. 45]) for each $k \geq 2$ there exists $\Omega_{k} \subset \Omega$ with $\lambda\left(\Omega_{k}\right)=1$ such that $\frac{1}{n} \sum_{j=0}^{n-1} g_{k}\left(T^{j} \omega\right) \rightarrow E_{P} g_{k}$ for every $\omega \in \Omega_{k}$. Let $\omega \in \bigcap_{k \geq 2} \Omega_{k}$ and observe that

$$
\begin{aligned}
\left|\frac{1}{n} \sum_{j=1}^{n} f\left(u_{j}\right)-m\right| \leq & \frac{1}{n}\left|\sum_{j=1}^{k-1} f\left(u_{j}\right)\right|+\frac{1}{n} \sum_{j=k}^{n}\left|f\left(u_{j}\right)-g_{k}\left(T^{j-k}\right)\right| \\
& +\left|\frac{1}{n} \sum_{j=k}^{n} g_{k}\left(T^{j-k}\right)-E_{P} g_{k}\right|+\left|E_{P} g_{k}-m\right| .
\end{aligned}
$$

Using that $g_{k}\left(T^{j-k}\right)=f\left(\left[a_{j}, \ldots, a_{j+k}\right]+\left[0, a_{j-1}, \ldots, a_{j-(k-1)}\right]\right),\left|x_{j}-\left[a_{j}, \ldots, a_{j+k}\right]\right|$ $\leq 2^{-k+1}$ and $\mid y_{j-1}^{-1}-\left[0, a_{j-1}, \ldots, a_{j-(k-1)}\right] \leq 2^{-k+2}$ we see that the second term on the right is bounded by $6 K 2^{-k}$. The third term tends to 0 since $\omega \in \Omega_{k}$. Therefore, for every $k \geq 2$

$$
\varlimsup_{n \rightarrow \infty}\left|\frac{1}{n} \sum_{1}^{n} f\left(u_{j}\right)-m\right| \leq \frac{6 K}{2^{k}}+\left|E_{P} g_{k}-m\right|
$$

It suffices to observe that $E_{P} g_{k} \rightarrow m$ as $k \rightarrow \infty$ (note that $\left|E_{P} g_{k}-E_{P} f\left(u_{k}\right)\right| \leq$ $K 2^{-k+1}$ and that from (3.1) we can conclude, as in [18, p. 76], for example, that $\left|E_{P} f\left(u_{k}\right)-m\right|=O\left(s^{k}\right)$ for some $\left.s \in(0,1)\right)$.

Proof of Proposition 1.4. (Sketch) In [17, Chapter 7] take $\Omega$ endowed with $\lambda$ as the probability space, $\eta_{n}=f\left(u_{n}\right)-E f\left(u_{n}\right)$ (this $f$ plays a different role than the $f$ there), $\mathcal{F}_{i}^{j}$ the $\sigma$-field generated by $a_{i}, \ldots, a_{j}(1 \leq i \leq j)$ and $\eta_{n \ell}=E\left[\eta_{n} \mid \mathcal{M}_{n \ell}\right]$ with $\mathcal{M}_{n \ell}$ defined as in the proof of Lemma 1.2 (if $n>\ell, \mathcal{M}_{n \ell}=\mathcal{F}_{n-\ell}^{n+\ell}$ ). It is well known that $\left\{a_{j}\right\}$ is a $\psi$-mixing sequence of r.v.'s with exponential mixing rate (see, for example, [10], [8] or [6], where good bounds for the mixing coefficients are also proved); thus for any $\delta>0,(7.1 .2)$ of [17] is satisfied with $\beta(s)=s^{-168(1+2 / \delta)}$ and $\kappa$ given by (7.1.8).

Suppose that $f$ satisfies (1.2) and that $\sigma^{2}>0$ in Lemma 1.2. We can assume that $0<\delta \leq 2$. It is easy to show that (7.1.5) of [17] holds. Relation (3.8) implies $[17,(7.1 .6)]$. Finally, replace $[17,(7.1 .7)]$ by the equality of Lemma 1.2 which leds to consider $\{X(\sigma t)\}$ in place of $\{X(t)\}$. Then, with minor modifications, the proof given by Philipp and Stout works in the present situation. We only make two comments: in the proof of [17, Lemma 7.3.1] we will have, for some constants $C$ and $C^{\prime}$,

$$
\begin{aligned}
\rho(n, t) \leq & \beta\left(\left(\left[t^{47 \alpha}\right]+1-\left[t^{46 \alpha}\right]+\left[(t+n-1)^{47 \alpha}\right]-\left[(t+n)^{46 \alpha}\right]\right.\right. \\
& \left.\left.+\sum_{j=t+1}^{t+n-2}\left[j^{47 \alpha}\right]+\sum_{j=t+1}^{t+n-1}\left[j^{100 \alpha}\right]\right)\left(C t^{100 \alpha+1}\right)^{-\frac{\delta}{11+4 \delta}}\right) \\
\leq & \left(C^{\prime}\left(t^{47 \alpha}+(t+n-1)^{100 \alpha+1}-t^{100 \alpha+1}\right) t^{-42 \alpha}\right)^{-168\left(1+\frac{2}{\delta}\right)}
\end{aligned}
$$

the proof of [17, Lemma 7.3.3] contains a mistake and is corrected in the Errata (the Borel-Cantelli lemma and Markov's inequality involving $2+\delta$-moments are 
used in order to prove, in place of (7.3.4), that $Q\left(M_{N}\right) \ll M_{N}^{\frac{1}{2}+47 \alpha}$ a.s.). At the end use (3.1) to replace $E f\left(u_{n}\right)$ by $m$.

For the case of $f$ as in (1.1) (and $\eta_{n}$ defined with $u_{n}$ ) we take $\delta=2$ and observe that arguing as for $(3.9)$ we have $\left\|\eta_{j}-E\left[\eta_{j} \mid \mathcal{M}_{j \ell}\right]\right\|_{4} \leq C^{\frac{1}{4}} \frac{1}{\left(2^{\frac{1}{4}}\right)^{\ell}}$ which implies [17, (7.1.6)]. The proof for $\left\{y_{j}\right\}$ is similar (use (3.16) or (3.17)).

Proof of Corollary 1.5. a) Since $\sigma^{2}=0, \sup _{n \geq 1} \operatorname{ES}^{2}(n)<\infty$ by Lemma 1.2 and its proof. Hence $\sum_{n} \lambda\left(\left|r(n)^{-1} S(n)\right|>\varepsilon\right)<\infty$ for every $\varepsilon>0$ (each term is $\ll \varepsilon^{-2} r(n)^{-2}$ ), which implies the conclusion by the Borel-Cantelli lemma.

b) We adapt an argument of Rajchman [4, Theorem 5.1.2]. Write $\gamma=1+\varepsilon$. Let $r(n)=(\log n)^{\gamma}, t(n)=\left[e^{n^{1 / \gamma}}\right]+1$ and

$$
D_{n}=\max _{t(n)<k \leq t(n+1)}|S(k)-S(t(n))| .
$$

If $t(n)<k \leq t(n+1)$ we have

$$
\frac{1}{r(k)}|S(k)| \leq \frac{1}{n}|S(t(n))|+\frac{1}{n} D_{n} .
$$

Therefore it is sufficient to show that both terms on the right-hand member tend to 0 a.e. as $n \rightarrow \infty$. For the first term we get the result as in a). For the second one we will show again that $\sum_{n} \lambda\left(\left|\frac{1}{n} D_{n}\right|>\delta\right)<\infty$ for every $\delta>0$.

Fix $\delta>0$ and consider $\eta_{n j}:=\frac{1}{n} \eta_{j}, j=t(n)+1, \ldots, t(n+1), n \geq 1$, where the $\eta_{j}$ 's are the terms in $S(n)$, which are bounded by, say, $M$. We will use the maximal inequality in [18, Corollary 3.1], taken from Billingsley [2]; it is stated in terms of the Gauss' measure $P$ and involves the mixing coefficients $\phi(k)$ of $\left\{a_{j}\right\}$, which satisfy $\phi(k) \ll \rho_{1}^{k}$ for some $\rho_{1} \in(0,1)$. Take $p_{n}=\left[n^{1 / \beta}\right]$ with $1<\beta<\gamma$. Note that by the proof of Lemma 1.2 we get $\left\|\eta_{n j}-E\left[\eta_{n j} \mid \mathcal{M}_{j \ell}\right]\right\|_{2} \ll \rho_{2}^{\ell} / n$ for some $\rho_{2} \in(0,1)$, where the expectations are now taken with respect to $P$. Then for all sufficiently large $n, p_{n} \leq(t(n+1)-t(n)) / 2$ and the quoted inequality gives (for our fixed $\delta$ )

$$
\begin{aligned}
\lambda\left(\left|\frac{1}{n} D_{n}\right|>\delta\right) & \ll \rho_{1}^{2 n^{1 / \beta}}+(t(n+1)-t(n)) \frac{1}{n^{2}} \rho_{2}^{2 n^{1 / \beta}}+\frac{1}{n^{2}} \\
& \ll \rho_{1}^{2 n^{1 / \beta}}+e^{n^{1 / \gamma}} n^{1 / \gamma-3} \rho_{2}^{2 n^{1 / \beta}}+\frac{1}{n^{2}}
\end{aligned}
$$

because $\max _{0 \leq k \leq t(n+1)-t(n)-2 p_{n}} P\left(\sum_{j=t(n)+k+1}^{t(n)+k+2 p_{n}}\left|\eta_{n j}\right|>\delta\right)=0$ for $n$ such that $2 M p_{n} / n \leq \delta$. This shows the convergence of the series and proves $\mathrm{b}$ ).

d) follows from b) when $\sigma^{2}=0$ and from Proposition 1.4 when $\sigma^{2}>0$ since it implies the law of the iterated logarithm (see [17, Theorem C]). Proposition 1.4 and a) imply c).

Proof of Lemma 1.6. Assume $f$ satisfies (1.2) with $0<\delta \leq 1$. We consider the r.v.'s $\eta_{j}=f\left(u_{j}\right)-E f\left(u_{j}\right)$ and introduce the notation: $S_{0}(h)=0, S_{n}(h)=\sum_{j=h+1}^{h+n} \eta_{j}$ if $n \geq 1, h \geq 0 ; A_{n}(h)=E\left|S_{n}(h)\right|^{2+\delta}(n \geq 0, h \geq 0) ; A_{n}=\sup _{h \geq 0} A_{n}(h)(n \geq 0)$. Note that $A_{1}<\infty$ and $A_{n} \leq A_{1} n^{2+\delta}$ for each $n$. By (3.1) it suffices to prove that

$$
\sup _{n \geq 1, h \geq 0} E\left(\left|\frac{1}{\sqrt{n}} S_{n}(h)\right|^{2+\delta}\right)<\infty .
$$


We will argue as in the proof of [10, Lemma 18.5.1]. We claim that we have the following analogue of $[10,(18.5 .1)]$ :

(A) for every $\varepsilon_{1}>0$ there exist $C_{1}$ and $k \geq 1$ such that

$$
\forall n \geq 1, \forall h \geq 0, E\left(\left|S_{n}(h)+S_{n}(h+n+k)\right|^{2+\delta}\right) \leq\left(2+\varepsilon_{1}\right) A_{n}+C_{1} n^{1+\frac{\delta}{2}} .
$$

First observe that one can argue as in the proof of Lemma 1.2 to show that for some constant $C$

$$
\sup _{h \geq 0} E\left(\left(S_{n}(h)\right)^{2}\right) \leq C n \text { for every } n \text {. }
$$

Fix $k \geq 1$. Given $n \geq 1, h \geq 0$ write $S_{n}=S_{n}(h)$ and $\hat{S}_{n}=S_{n}(h+n+k)$. Since $\delta \leq 1$

$$
\begin{aligned}
E\left|S_{n}+\hat{S}_{n}\right|^{2+\delta} & \leq E\left(\left(\left|S_{n}\right|^{2}+2\left|S_{n}\right|\left|\hat{S}_{n}\right|+\left|\hat{S}_{n}\right|^{2}\right)\left(\left|S_{n}\right|^{\delta}+\left|\hat{S}_{n}\right|^{\delta}\right)\right) \\
& \leq 2 A_{n}+E\left(\left|S_{n}\right|^{2}\left|\hat{S}_{n}\right|^{\delta}\right)+2 E\left(\left|S_{n}\right|^{1+\delta}\left|\hat{S}_{n}\right|\right) \\
& +2 E\left(\left|S_{n}\right|\left|\hat{S}_{n}\right|^{1+\delta}\right)+E\left(\left|S_{n}\right|^{\delta}\left|\hat{S}_{n}\right|^{2}\right) \\
& =2 A_{n}+I_{1}+2 I_{2}+2 I_{3}+I_{4} \text { (say). }
\end{aligned}
$$

Put $\mathcal{F}=\mathcal{F}_{1}^{h+n}, \mathcal{G}=\mathcal{F}_{h+n+k}^{h+n+k+2 n}$ where $\mathcal{F}_{i}^{j}$ is as in the proof of Proposition 1.4. Given positive integers $i_{h+n+k}, \ldots, i_{h+n+k+2 n}$, if $\omega, \omega^{\prime} \in \Delta:=\left\{a_{h+n+k}=\right.$ $\left.i_{h+n+k}, \ldots, a_{h+n+k+2 n}=i_{h+n+k+2 n}\right\}$ then

$$
\begin{aligned}
\left.|| \hat{S}_{n}(\omega)\right|^{\delta}-\left|\hat{S}_{n}\left(\omega^{\prime}\right)\right|^{\delta} \mid & \leq\left|\hat{S}_{n}(\omega)-\hat{S}_{n}\left(\omega^{\prime}\right)\right|^{\delta} \\
& \leq \sum_{j=h+n+k+1}^{h+n+k+n}\left|f\left(u_{j}(\omega)\right)-f\left(u_{j}\left(\omega^{\prime}\right)\right)\right|^{\delta} \\
& \leq K \sum_{j=h+n+k+1}^{h+n+k+n}\left(6 \times 2^{-(j-(h+n+k))}\right)^{\delta} \leq K_{1}
\end{aligned}
$$

for some constant $K_{1}$. Hence for every $\omega \in \Omega$, if $\Delta$ is the unique set of the above form such that $\omega \in \Delta$,

$$
\left.|| \hat{S}_{n}(\omega)\right|^{\delta}-\frac{1}{\lambda(\Delta)} \int_{\Delta}\left|\hat{S}_{n}\right|^{\delta} d \lambda|=| \frac{1}{\lambda(\Delta)} \int_{\Delta}\left\{\left|\hat{S}_{n}(\omega)\right|^{\delta}-\left|\hat{S}_{n}\right|^{\delta}\right\} d \lambda \mid \leq K_{1} .
$$

Thus

$$
\left.|| \hat{S}_{n}\right|^{\delta}-E\left[\left|\hat{S}_{n}\right|^{\delta} \mid \mathcal{G}\right] \mid \leq K_{1} \quad \text { a.s. }
$$

Similarly if $\omega, \omega^{\prime} \in \Delta:=\left\{a_{1}=i_{1}, \ldots, a_{h+n}=i_{h+n}\right\},\left.|| S_{n}(\omega)\right|^{2}-\left|S_{n}\left(\omega^{\prime}\right)\right|^{2} \mid \leq$ $K^{\prime}{ }_{1}\left\{\left|S_{n}(\omega)\right|+\left|S_{n}\left(\omega^{\prime}\right)\right|\right\}$; this implies

$$
\left.|| S_{n}\right|^{2}-E\left[\left|S_{n}\right|^{2} \mid \mathcal{F}\right] \mid \leq K_{1}^{\prime}\left\{\left|S_{n}\right|+E\left[\left|S_{n}\right| \mid \mathcal{F}\right]\right\} \quad \text { a.s. }
$$


Also we have:

$$
\begin{gathered}
|| \hat{S}_{n}\left|-E\left[\left|\hat{S}_{n}\right| \mid \mathcal{G}\right]\right| \leq K_{2} \text { a.s., } \\
\left.|| S_{n}\right|^{1+\delta}-E\left[\left|S_{n}\right|^{1+\delta} \mid \mathcal{F}\right] \mid \leq K_{2}^{\prime}\left\{\left|S_{n}\right|^{\delta}+E\left[\left|S_{n}\right|^{\delta} \mid \mathcal{F}\right]\right\} \quad \text { a.s. } \\
\left.|| \hat{S}_{n}\right|^{1+\delta}-E\left[\left|\hat{S}_{n}\right|^{1+\delta} \mid \mathcal{G}\right] \mid \leq K_{3}\left\{\left|\hat{S}_{n}\right|^{\delta}+E\left[\left|\hat{S}_{n}\right|^{\delta} \mid \mathcal{G}\right]\right\} \quad \text { a.s. } \\
|| S_{n}\left|-E\left[\mid S_{n} \| \mathcal{F}\right]\right| \leq K_{3}^{\prime} \text { a.s., } \\
\left.|| \hat{S}_{n}\right|^{2}-E\left[\left|\hat{S}_{n}\right|^{2} \mid \mathcal{G}\right] \mid \leq K_{4}\left\{\left|\hat{S}_{n}\right|+E\left[\left|\hat{S}_{n}\right| \mid \mathcal{G}\right]\right\} \quad \text { a.s. }
\end{gathered}
$$

and

$$
\left.|| S_{n}\right|^{\delta}-E\left[\left|S_{n}\right|^{\delta} \mid \mathcal{F}\right] \mid \leq K_{4}^{\prime} \text { a.s. }
$$

Now observe that

$$
\begin{aligned}
I_{1} & \leq E\left\{E\left[\left|S_{n}\right|^{2} \mid \mathcal{F}\right] \times E\left[\left|\hat{S}_{n}\right|^{\delta} \mid \mathcal{G}\right]\right\}+E\left\{E\left[\left|S_{n}\right|^{2} \mid \mathcal{F}\right] \times\left.|| \hat{S}_{n}\right|^{\delta}-E\left[\left|\hat{S}_{n}\right|^{\delta} \mid \mathcal{G}\right] \mid\right\} \\
& +E\left\{\left.|| S_{n}\right|^{2}-E\left[\left|S_{n}\right|^{2} \mid \mathcal{F}\right] \mid \times E\left[\left|\hat{S}_{n}\right|^{\delta} \mid \mathcal{G}\right]\right\} \\
& +E\left\{\left.|| S_{n}\right|^{2}-E\left[\left|S_{n}\right|^{2} \mid \mathcal{F}\right]|\times|\left|\hat{S}_{n}\right|^{\delta}-E\left[\left|\hat{S}_{n}\right|^{\delta} \mid \mathcal{G}\right] \mid\right\} \\
& =I_{1}^{(1)}+I_{1}^{(2)}+I_{1}^{(3)}+I_{1}^{(4)} \text { (say). }
\end{aligned}
$$

If $\phi$ denotes the mixing coefficient of $\left\{a_{j}\right\}$ (under $\lambda$ ), using an inequality of Ibragimov ([10, Theorem 17.2.3], which does not require stationarity, with $p=$ $\left.\frac{2+\delta}{2}, q=\frac{2+\delta}{\delta}\right)$, Jensen's inequality and (3.23) we obtain

$$
I_{1}^{(1)} \leq 2(\phi(k))^{\frac{2}{2+\delta}} A_{n}+C^{1+\frac{\delta}{2}} n^{1+\frac{\delta}{2}} .
$$

From (3.24)-(3.25) we get $I_{1}^{(2)} \leq K_{1} C n, I_{1}^{(3)} \leq 2 K_{1}^{\prime} C^{(1+\delta) / 2} n^{(1+\delta) / 2}$ and $I_{1}^{(4)} \leq$ $2 K_{1} K_{1}^{\prime} C^{1 / 2} n^{1 / 2}$. Then $I_{1} \leq 2(\phi(k))^{2 /(2+\delta)} A_{n}+C_{1}^{\prime} n^{1+\delta / 2}$.

Using (3.26)-(3.27) one can prove that $I_{2} \leq 2(\phi(k))^{(1+\delta) /(2+\delta)} A_{n}+C_{2}^{\prime} n^{1+\delta / 2}$; (3.28)-(3.29) imply that $I_{3} \leq 2(\phi(k))^{1 /(2+\delta)} A_{n}+C_{3}^{\prime} n^{1+\delta / 2}$ and (3.30)-(3.31) give that $I_{4} \leq 2(\phi(k))^{\delta /(2+\delta)} A_{n}+C_{4}^{\prime} n^{1+\delta / 2}$. By the $\phi$-mixing property of $\left\{a_{j}\right\}$, an appropriate choice of $k$ completes the proof of (A).

The assertion

(B) for every $\varepsilon_{2}>0$ there exists $C_{2}$ such that

$$
\forall n \geq 1, A_{2 n} \leq\left(2+\varepsilon_{2}\right) A_{n}+C_{2} n^{1+\frac{\delta}{2}}
$$

is a consequence of $(\mathrm{A})$ (see [10]). By induction we can prove:

$$
\forall r \geq 1, A_{2^{r}} \leq\left(2+\varepsilon_{2}\right)^{r} A_{1}+C_{2} \sum_{j=1}^{r}\left(2+\varepsilon_{2}\right)^{j-1}\left(2^{r-j}\right)^{1+\frac{\delta}{2}} .
$$

We can complete the proof by considering $\varepsilon_{2}>0$ such that $\gamma:=\left(2+\varepsilon_{2}\right) 2^{-(1+\delta / 2)}$ $<1$.

The proof for $y_{j}$ and $x_{j}$ is analogous. 


\section{Remarks on the CONdition $\sigma^{2}>0\left(x_{j}\right.$ CASE $)$}

For $S(t)=\sum_{1 \leq j \leq t}\left(f\left(x_{j}\right)-m\right)$, with $m$ defined in (3.20), the work by Gordin [6] on the transformation $T$ gives useful sufficient conditions for the strict positivity of $\sigma^{2}$ in Lemma 1.2. For example, one can take $f(x)=\frac{1}{x}$ and $f(x)=\log x$ (the first case follows from the facts discussed in b) on [6, p. 480] and the second is solved in [6, p. 481]; alternatively, see 4.2 below); this last choice gives then, as a direct consequence of [17, Theorem 7.1], an almost sure invariance principle for $\log q_{n}$ because $\left\{\log q_{n}-\sum_{1}^{n} \log x_{j}\right\}$ is bounded (see [6], [2, p. 193]; this improves [9], the functional limit theorem for $[2,(21.52)]$, one of the theorems in [16] and [7]), which in turn gives the same result for $-\log d_{n}$ where $d_{n}(\omega):=\left|q_{n}(\omega) \cdot \omega-p_{n}(\omega)\right|$ (see [2, pp. 193-194]).

We will show that in the case of $x_{j}$ we also have $\sigma^{2}>0$ for $f$ as in (1.1). This is not a direct corollary of the criteria in [6] (unless $b$ is an integer, in which case $f\left(x_{j}\right)$ reduces to $\left.f\left(a_{j}\right)\right)$; it follows from our Lemma 4.1, another criterion derived from the work of Gordin, which also covers the above examples (see 4.2). Hence one has that $\sigma^{2}>0$ in the functional CLT for the indicator function in [2, p. 193] and the almost sure invariance principle also holds in this case.

Now we consider $T$ and Gauss' measure $P$ defined on $[0,1]\left(T \omega=\frac{1}{\omega}-\left[\frac{1}{\omega}\right]\right.$ if $\omega \neq 0, T 0=0)$.

4.1. Lemma. Assume $\tilde{f}:[0,1] \longrightarrow \mathbb{R}$ is continuous except for a finite number of points, $\tilde{f}$ is integrable with $E_{P} \tilde{f}=0$, the series

$$
(V \tilde{f})(x)=\sum_{k=1}^{\infty} \frac{x+1}{(x+k)(x+k+1)} \tilde{f}\left(\frac{1}{k+x}\right)
$$

converges uniformly on $[0,1]$ giving a function of bounded variation and

$$
\inf _{x \in(0, \delta)}|\tilde{f}(x)|>0 \quad \text { for some } \delta>0 .
$$

Then there exists no $g \in L^{2}([0,1], P)$ such that $E_{P} g=0$ and $\tilde{f}(x)=g(T x)-g(x)$ for almost every $x$.

Proof. Assume that there exists $g \in L^{2}$ with zero expectation satisfying the equation. Then $\left(\left[6\right.\right.$,Theorem 3]) we can take as $g(x)$ the function $\sum_{k=1}^{\infty}\left(V^{k} \tilde{f}\right)(x)$, the series converging uniformly on $[0,1]$ by relation $(9)$ in $[6]$.

Now observe that if $h$ is a function continuous in $[0,1]$ except for the points in a finite set $D$, say, and the series defining $V h$ converges uniformly, then $V h$ also has a finite number of discontinuities. In fact, $V h$ is continuous at $x$ if $x \notin T(D) \cup\{1\}$ (if $k>k_{0}:=\max \{[1 / d]: d \in D\}$, the $k$ th term in (4.1), with $h$ in place of $\tilde{f}$, is continuous in $[0,1]$ since for $x \in[0,1], \frac{1}{k+x}=d$ is equivalent to $\left[\frac{1}{d}\right]=k$ or $\frac{1}{d}=k+1$; then the sum of those terms is continuous. If $k \leq k_{0}$ the set of those $x \in[0,1]$ such that $\frac{1}{k+x} \in D$ consists of the points $T d, d \in D \cap\left(\frac{1}{k+1}, \frac{1}{k}\right]$, and, eventually, 1 (if $\left.d=\frac{1}{k+1}\right)$; hence the $k$ th term is continuous at $\left.x \notin T(D) \cup\{1\}\right)$.

Let $0<\varepsilon<\frac{1}{3} \inf _{x \in(0, \delta)}|\tilde{f}(x)|$. By relation (9) in [6] there exists $k_{1}$ such that $g_{1}:=\sum_{k>k_{1}} V^{k} \tilde{f}$ satisfies $\left|g_{1}(x)\right| \leq \varepsilon$ for every $x \in[0,1]$; by the preceding observation the function $g_{2}:=\sum_{1 \leq k \leq k_{1}} V^{k} \tilde{f}$ has a finite number of points of discontinuity. 
Take $n \geq \frac{1}{\delta}$ such that $\tilde{f}$ and $g_{2}$ are continuous in $\left(\frac{1}{n+1}, \frac{1}{n}\right)$ and let $t^{(n)}$ be the fixed point of $T$ in this interval. Since the equation is assumed to hold a.e. there exists a sequence $\left\{t_{j}\right\} \subset\left(\frac{1}{n+1}, \frac{1}{n}\right)$ such that $t_{j} \rightarrow t^{(n)}$ and $\tilde{f}\left(t_{j}\right)=g\left(T t_{j}\right)-g\left(t_{j}\right)$ for each $j$. We have $T t_{j}=\left(1 / t_{j}\right)-n \rightarrow\left(1 / t^{(n)}\right)-n=t^{(n)}$ and by the choice of $n$ we can thus obtain a $t_{j_{0}}$ such that $\left|g_{2}\left(T t_{j_{0}}\right)-g_{2}\left(t_{j_{0}}\right)\right| \leq \varepsilon$. But this implies that $\left|g\left(T t_{j_{0}}\right)-g\left(t_{j_{0}}\right)\right| \leq 3 \varepsilon<\left|\tilde{f}\left(t_{j_{0}}\right)\right|$.

4.2. Examples. The condition $\sigma^{2}>0$ is satisfied in the case of $x_{j}$ for $f$ as in (1.1) and if $f:[1, \infty) \longrightarrow \mathbb{R}$ is a Lipschitz function such that $f(x)=O\left(x^{\alpha}\right)$ as $x \rightarrow \infty$ with $\alpha<\frac{1}{2}$ (then $f$ satisfies (1.2)) and $\inf _{x>x_{0}}|f(x)-m|>0$ for some $x_{0}$; for example if $f(x)=x^{\alpha}(\log x)^{\beta}$ with $\alpha<\frac{1}{2}$ and any $\beta$. In order to see this, observe that $\tilde{f}(\omega):=f\left(\frac{1}{\omega}\right)-m$ if $\omega \in(0,1],=0$ if $\omega=0$, satisfies the hypotheses of Lemma 4.1 (for the indicator, note that $m=P([0,1 / b])<1$ ) and use a theorem of Leonov $[6]$.

Acknowledgment. We thank Professor Walter Philipp for directing our attention to $[6]$.

\section{REFERENCES}

1. P. Billingsley, Ergodic Theory and Information, Wiley, New York, 1965. MR 33:254

2. P. Billingsley, Convergence of probability measures, Wiley, New York, 1968. MR 38:1718

3. W. Bosma, H. Jager and F. Wiedijk, Some metrical observations on the approximation by continued fractions, Indag. Math. 45 (1983), 281-299. MR 85f:11059

4. K. L. Chung, A course in Probability Theory, 2nd edition, Academic Press, New York, 1974. MR 49:11579

5. W. Doeblin, Remarques sur la théorie métrique des fractions continues, Compositio Math. 7 (1940), 353-371. MR 2:107e

6. M. I. Gordin, On the behavior of the variances of sums of random variables forming a stationary process, Theory Probab. Appl. 16 (1971), 474-484. MR 44:4809

7. M. I. Gordin and M. H. Reznik, The law of the iterated logarithm for the denominators of continued fractions, Vestnik Leningrad Univ. 25 (1970), 28-33. (Russian) MR 43:1939

8. S. Grigorescu and M. Iosifescu, Dependence with Complete Connections and its Applications, Cambridge University Press, Cambridge, 1990. MR 91j:60098

9. I. A. Ibragimov, A metrical theorem in the theory of continued fractions, Vestnik Leningrad Univ. 1 (1961), 13-24. (Russian) MR 24:A3445

10. I. A. Ibragimov and Yu. V. Linnik, Independent and Stationary Sequences of Random Variables, Wolters-Noordhoff, Groningen, 1971. MR 48:1287

11. M. Iosifescu, On mixing coefficients for the continued fraction expansion, Stud. Cerc. Mat. 41 (1989), 491-499. MR 91h:11079

12. D. E. Knuth, The Art of Computer Programming, Vol. 2. Seminumerical Algorithms, AddisonWesley, Reading, Mass., 1981. MR 83i:68003

13. D. E. Knuth, The distribution of continued fraction approximations, J. Number Theory 19 (1984), 443-448. MR 86d:11058

14. P. Lévy, Théorie de l'addition des variables aléatoires, Gauthier-Villars, Paris, 1954.

15. G. Misevičius, The evaluation of the remainder term in the limit theorem for functions of the elements of continued fractions, Lietuvos Matematikos Rinkinys 10 (1970), 293-308. (Russian) MR 45:5102

16. W. Philipp and O. P. Stackelberg, Zwei Grenzwertsätze für Kettenbrüche, Math. Ann. 181 (1969), 152-156. MR 39:5503

17. W. Philipp and W. Stout, Almost sure invariance principles for partial sums of weakly dependent random variables, Mem. Amer. Math. Soc. 161 (1975), Errata (preprint, 1975). MR 55:6570 
18. J. D. Samur, On some limit theorems for continued fractions, Trans. Amer. Math. Soc. 316 (1989), 53-79. MR 90b:60030

19. J. D. Samur, A functional central limit theorem in Diophantine approximation, Proc. Amer. Math. Soc. 111 (1991), 901-911. MR 91g:11087

Departamento de Matemática, Facultad de Ciencias Exactas, Universidad Nacional de La Plata, Casilla de Correo 172, 1900 La Plata, Argentina

E-mail address: jorge@mate.unlp.edu.ar 